\title{
Assessment of the interphotoreceptor matrix proteoglycan-1 (IMPG1) gene localised to 6q13-q15 in autosomal dominant Stargardt-like disease (ADSTGD), progressive bifocal chorioretinal atrophy (PBCRA), and North Carolina macular dystrophy (MCDR1)
}

\author{
Andrea Gehrig, Ute Felbor, Rosemary E Kelsell, David M Hunt, Irene H Maumenee, \\ Bernhard H F Weber
}

Institut für

Humangenetik,

Biozentrum,

Universität Würzburg,

Am Hubland, D-97074,

Germany

A Gehrig

U Felbor

B H F Weber

Augenklinik,

Universität Würzburg,

D-97074 Würzburg, Germany

U Felbor

Institute of

Ophthalmology,

University College

London, London

EC1V 9EL, UK

R E Kelsell

D M Hunt

The Wilmer Eye Institute, The Johns Hopkins Center for Hereditary Eye

Diseases, Baltimore,

MD 21287-9237, USA

I H Maumenee

Correspondence to:

Dr Weber.

Received 14 October 1997 Revised version accepted for publication 12 January 1997

\begin{abstract}
We have recently characterised the genomic organisation of a novel interphotoreceptor matrix proteoglycan, IMPG1, and have mapped the gene locus to chromosome 6q13-q15 by fluorescence in situ hybridisation. As the interphotoreceptor matrix (IPM) is thought to play a critical role in retinal adhesion and the maintenance of photoreceptor cells, it is conceivable that a defect in one of the IPM components may cause degenerative lesions in retinal structures and thus may be associated with human retinopathies. By genetic linkage analysis, several retinal dystrophies including one form of autosomal dominant Stargardt-like macular dystrophy (STGD3), progressive bifocal chorioretinal atrophy (PBCRA), and North Carolina macular dystrophy (MCDR1) have previously been localised to a region on proximal $6 \mathrm{q}$ that overlaps the IMPG1 locus. We have therefore assessed the entire coding region of IMPG1 by exon amplification and subsequent single stranded conformational analysis in patients from 6q linked multigeneration families diagnosed with PBCRA and MCDR1, as well as a single patient from an autosomal dominant STGD pedigree unlinked to either of the two known STGD2 and STGD3 loci on chromosomes 13q and 6q, respectively. No disease associated mutations were identified. In addition, using an intragenic polymorphism, IMPG1 was excluded by genetic recombination from both the PBCRA and the MCDR1 loci. However, as the autosomal dominant Stargardt-like macular dystrophies are genetically heterogeneous, other forms of this disorder, in particular STGD3 previously linked to $6 \mathrm{q}$, may be caused by mutations in IMPG1.

(F Med Genet 1998;35:641-645)
\end{abstract}

Keywords: Stargardt-like macular dystrophy; MCDR1; PBCRA; interphotoreceptor matrix proteoglycan-1

The positional candidate approach relies on the mapping of a disease locus and a candidate gene to a common chromosomal region and the assessment of the functional properties of the gene product with regard to the disease pathology. Several criteria, such as tissue specificity of gene expression, cellular localisation of the transcript, immunohistochemical data, possibly in combination with interspecies nucleotide/ protein sequence comparison or protein pattern database searches, may provide helpful information on the probable function of a candidate gene. With an increasing number of mapped genes and expressed sequence tags (ESTs) available through the international efforts of the Human Genome Project, the positional candidate strategy has become the method of choice for the identification of disease genes.

Recently, a novel gene, IMPG1, encoding a major proteoglycan of the interphotoreceptor matrix (IPM), was identified and shown to be preferentially expressed in retina by both rod and cone photoreceptor cells. ${ }^{23}$ The IPM occupies the space between the neurosensory retina and the retinal pigment epithelium (RPE) and is largely composed of insoluble glycoconjugates that appear to form specific structures around the rod and cone photoreceptor cells, commonly referred to as cone matrix sheaths and rod matrix domains. ${ }^{47}$ The IPM glycoconjugates, which are primarily chondroitin sulphate proteoglycans, have been proposed to mediate interactions among various cell types, including the RPE, the photoreceptors, the glial Müller cells, and the choriocapillaris ${ }^{89}$ and may be vital for retinal adhesion and maintenance of photoreceptor cells. ${ }^{111}$

More recently, we have determined the genomic organisation of IMPG1 and have mapped the gene locus to chromosomal region $6 \mathrm{q} 13-\mathrm{q} 15^{12}$ (fig 1). Interestingly, several retinal dystrophies including autosomal dominant Stargardt-like macular dystrophy (STGD3), ${ }^{13}$ progressive bifocal chorioretinal atrophy (PBCRA), ${ }^{14}$ and North Carolina macular dystrophy (MCDR1) ${ }^{15}$ have been localised to an overlapping region on the proximal long arm of chromosome 6 by genetic linkage analysis (fig 1). In order to assess whether mutations in IMPG1 may be associated with one of these macular dystrophies, we have now analysed the 17 coding exons of the gene for disease causing mutations in a family with autosomal dominant Stargardt-like macular dystrophy (ADSTGD), 


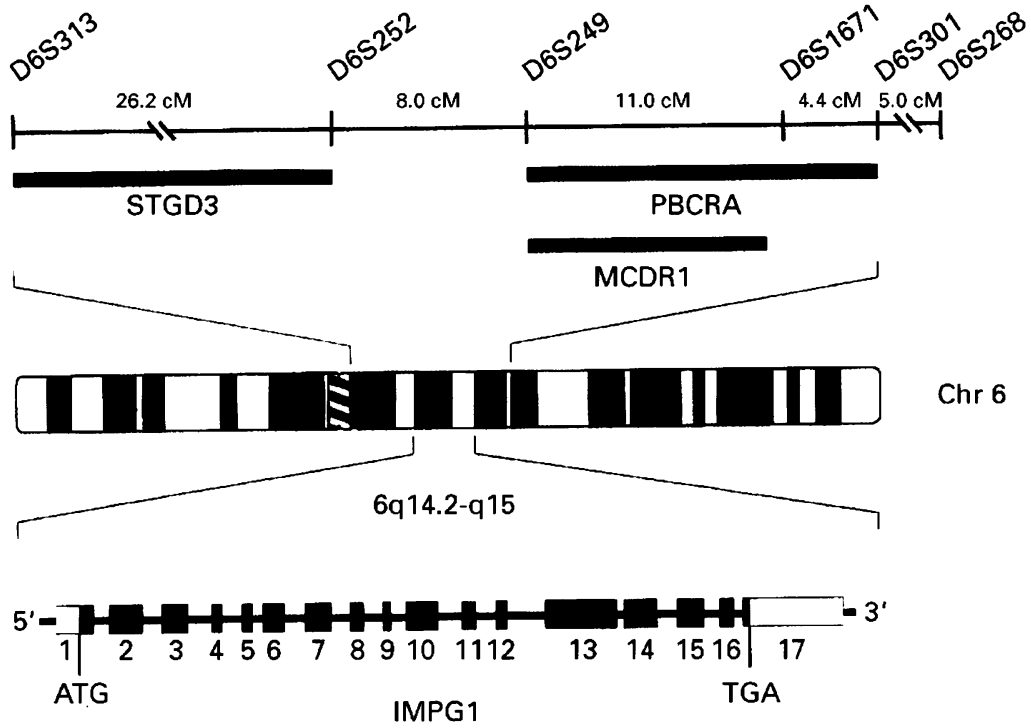

Figure 1 Idiogram of chromosome 6 and genetic map of proximal $6 q$ illustrating the relative locations and flanking DNA markers of gene loci for autosomal dominant Stargardt-like macular dystrophy (STGD3), ${ }^{13}$ North Carolina macular dystrophy (MCDR1), ${ }^{15}$ and progressive bifocal chorioretinal atrophy (PBCRA). ${ }^{14}$ Below, the genomic organisation of the gene encoding the interphotoreceptor matrix proteoglycan-1 (IMPG1) and its chromosomal localisation is shown. ${ }^{2}$

so far unlinked to STGD2 or STGD $3,{ }^{16}$ in three MCDR1 pedigrees of German descent recently linked to proximal $6 \mathrm{q}^{17}{ }^{18}$ and in a five generation British PBCRA pedigree previously mapped to proximal $6 \mathrm{q} .{ }^{14}$ Our results definitively exclude IMPG1 as a candidate gene for MCDR1 and PBCRA on the basis of SSCA and the analysis of recombinant chromosomes. Furthermore, direct sequencing has not shown any disease associated mutations in our patient with ADSTGD. However, as autosomal dominant Stargardt-like macular dystrophies are known to be genetically heterogeneous, ${ }^{131619}$ an involvement of IMPG1 in one form of ADSTGD cannot be ruled out at present and must await the mutational analyses

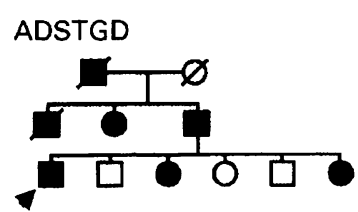

MCDR1-L

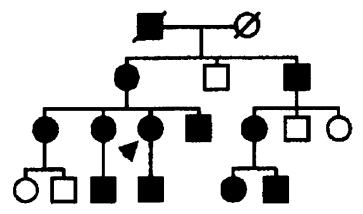

MCDR1-A

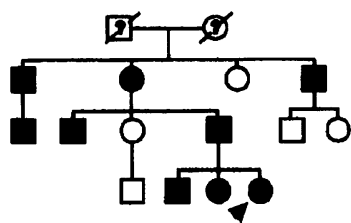

MCDR1-W

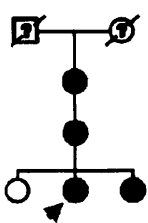

PBCRA

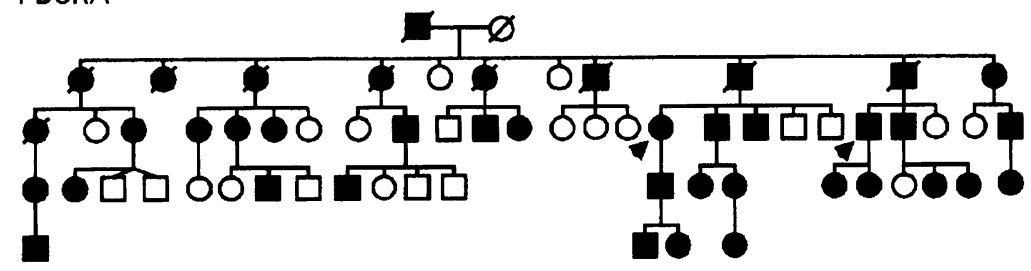

Figure 2 Pedigrees segregating autosomal dominant Stargardt-like macular dystrophy (ADSTGD), ${ }^{16}$ North Carolina macular dystrophy (MCDR1), 15 and progressive bifocal chorioretinal atrophy (PBCRA)." The closed symbols indicate affected status, while open symbols indicate subjects who were examined and found to be clinically normal. Affected subjects analysed for mutations in the IMPG1 gene are marked by an arrow. of the IMPG1 gene in STDG3 previously linked to proximal $6 \mathrm{q} .{ }^{13}$ The availability of the exon/ intron structure will allow the further assessment of the potential role of IMPG1 in various retinopathies of unknown aetiology.

\section{Materials and methods}

PATIENTS AND FAMILIES

The clinical, histopathological, and ultrastructural phenotype of a three generation pedigree with autosomal dominant Stargardt-like macular dystrophy (also known as fundus flavimaculatus-like macular dystrophy) and late onset atrophic macular degeneration has been described in detail elsewhere. ${ }^{16}$ In brief, mild blurring of central vision was first noted by the index patient at the age of 38. A paracentric scotoma was present in both eyes. Ophthalmoscopy showed a reddish foveal appearance surrounded by small yellowish-white, atrophic subretinal flecks and a reticular pattern of subretinal pigmentation in the midperiphery of both eyes. Multiple focal and confluent areas of choroidal hyperfluorescence were detected by fluorescein angiography. Bilateral retinal pigment epithelium (RPE) window defects were present in the fovea. There were areas of silent or dark choroid. The photopic electroretinogram (ERG) was subnormal in both eyes whereas scotopic ERG and electro-oculogram (EOG) were within the normal range. Inspection of the pedigree indicates autosomal dominant inheritance (fig 2). For mutational analysis, a DNA sample from a single affected subject was available (arrowed in fig 2).

In a recent study, the North Carolina macular dystrophy (MCDR1) phenotype was linked in three multigeneration families of German descent to DNA markers derived from chromosome 6q14-q16.2, ${ }^{17}$ a region known to harbour the MCDR1 gene. ${ }^{15}$ The family members underwent comprehensive ophthalmological examination. The affected subjects in families $\mathrm{L}, \mathrm{A}$, and $\mathrm{W}$ presented with fundus abnormalities consistent with MCDR1 and are described in detail elsewhere. ${ }^{17} 18$ The DNA from one affected subject per family was used for the mutational analyses (arrowed in fig 2).

Genetic linkage analysis on a five generation British pedigree expressing the progressive bifocal chorioretinal atrophy (PBCRA) phenotype has mapped the gene locus by two point linkage to an interval between D6S249 and D6S301 on proximal chromosome 6q. ${ }^{14} \mathrm{De}-$ tailed descriptions of ophthalmological findings in individual members of the PBCRA family are given in Douglas et $a l^{20}$ and Godley et al. ${ }^{21}$ Briefly, PBCRA is a progressive disease characterised by two distinct foci of atrophy, a temporal focus which is present at birth and a nasal focus which appears in the second decade of life. In addition, it is associated with nystagmus, myopia, and a significant reduction in visual acuity and colour vision. Photopsia and retinal detachment are complications of the disease.

MUTATIONAL ANALYSIS

DNA was isolated from leucocyte nuclei by standard extraction methods. Based on the genomic exon/intron sequences of IMPG $1,{ }^{12}$ 
Table 1 Exon/intron oligonucleotide primers for IMPG1

\begin{tabular}{|c|c|c|c|c|c|}
\hline $\begin{array}{l}\text { Exon } \\
\text { No }\end{array}$ & $\begin{array}{l}\text { Exon size } \\
\text { (bp) }\end{array}$ & Forward primer & Reverse primer & $\begin{array}{l}\text { Fragment } \\
\text { size }(b p)\end{array}$ & $\begin{array}{l}\text { Annealing } \\
\left({ }^{\circ} \mathrm{C}\right)\end{array}$ \\
\hline 1 & 67 & 74: 5'-AGATTTGAGGTTGTTCTGTG-3' & 74R:5'-TCGGTAGATTTTATAGATAAGC-3' & 173 & 54 \\
\hline 2 & 234 & Ex2F: 5'-TATTCACCAGGATATCAATC-3' & 5'R: 5'-ATAACACAAGGCTAAACGG-3' & 303 & 51 \\
\hline 3 & 167 & 429F: 5'- GACCCTCTTAGGCATGGCC-3' & 429R: 5'-AACTGTTCGTGCAGTGTTCC-3' & 254 & 58 \\
\hline 4 & 29 & 605F: 5'-TCTTCGGTTGGCATTATCTC-3' & 605R: 5'-GAATAGCTTAGTTTCACTCC-3' & 165 & 53 \\
\hline 5 & 65 & 672F: 5'-TCTCTTTCACCACCTCTTCC-3' & 672R: 5'-TTGAATGAAAGTTGAGAAGC-3' & 150 & 51 \\
\hline 6 & 104 & 789F: 5'-CTCCATTCTCATTTTTGTGTC-3' & 789R: 5'-ATGAAGAGCTAGATCAACTC-3' & 182 & 53 \\
\hline 7 & 141 & 4F: 5'-GTTTAGGTTAACAGAAGTCC-3' & 4RII: 5'-CACCTCTCAGTCACAGCTTG-3' & $235^{\star}$ & 54 \\
\hline 8 & 59 & 947F: 5'-AGCAGAACCAAAGAGTATT-3' & 947R: 5'-AATTGAGAAGAGTTTGATTC-3' & 132 & 49 \\
\hline 9 & 21 & 993-1113F: 5'-TTGAATGTCTCAATAAATGTC-3' & 993-1113R: 5'-ATATAAAT TTGGGGGAGACG-3' & 151 & 51 \\
\hline 10 & 248 & Ex8F: 5'-TTTGGGTGGGGAATGTGTC-3' & Ex8R: 5'-TAAACTCAGAACTAAGCAATC-3' & 313 & 53 \\
\hline 11 & 77 & 1328F: 5'-AGGAAAGATACATTCAAATC-3' & 1328R: 5'-GGTTTTGAGACTTTGTTCC-3' & 167 & 49 \\
\hline 12 & 79 & 1399F: 5'-ACATCCT TTCTCAACATCTG-3' & 1399R: 5'-CATAGACTTTGTGAGGGGAA-3' & 146 & 53 \\
\hline 13 & 533 & 1F: 5'-TTGGGGAATACTTGGACATA-3' & 1880R: 5'-GAATTGTGAGCAGTGCCC-3' & $600+$ & 54 \\
\hline 14 & 220 & ExBF: 5'-AACACAGTTAAGAGGAAACC-3' & 2R: 5'-CACTTTCTGGGTTGGATTCT-3' & 280 & 53 \\
\hline 15 & 199 & 2214F: 5'-GCTATTCATACATGCGTTAA-3' & 3R: 5'-AATGAGTTCTTGAGTCTGTG-3' & 281 & 51 \\
\hline 16 & 73 & 2373F: 5'-AAGCCCAAGAAACAAGATAC-3' & 2373R: 5'-TGCAACCAACTTAGAAAGTA-3' & 145 & 51 \\
\hline 17 & 78 & 3'F: 5'-CCTTGGGTGGCATTTCTTA-3' & 2779R: 5'-AGAATTTACTGGTTGCCAAG-3' & 295 & 54 \\
\hline
\end{tabular}

^For SSCA: digestion with HinfI results in two fragments of 100 and $135 \mathrm{bp}$.

†For SSCA: digestion with NlaIV results in three fragments of 250,210 , and $140 \mathrm{bp}$.

oligonucleotide primer pairs were designed to amplify the 17 coding exons of the gene using the polymerase chain reaction (PCR) (table 1). Reaction mixtures contained approximately 50 ng template DNA, $15 \mathrm{pmol}$ of each primer, 100 $\mu \mathrm{mol} / 1$ dNTPs, $0.1 \mu \mathrm{l}\left[\alpha{ }^{32} \mathrm{P}\right] \mathrm{dCTP}(3000$ $\mathrm{Ci} / \mathrm{mmol}), 1 \times \mathrm{PCR}$ buffer supplemented with 1-2 mmol/1 $\mathrm{MgCl}_{2}$, and 0.5 units of Taq DNA polymerase. The reaction mixture was denatured once at $94^{\circ} \mathrm{C}$ for five minutes and was subsequently subjected to 32 cycles of $30 \mathrm{sec}-$ onds at $94^{\circ} \mathrm{C}, 30$ seconds at an annealing temperature optimised for each primer pair $\left(55-60^{\circ} \mathrm{C}\right)$, and 30 seconds at $72^{\circ} \mathrm{C}$, followed by a final extension at $72^{\circ} \mathrm{C}$ for five minutes. Five microlitres of the 1:9 diluted PCR samples were subsequently added to $95 \%$ formamide, 5 $\mathrm{mmol} / 1 \mathrm{NaOH}, 0.1 \%$ xylene cyanol, and $0.1 \%$ bromophenol blue. The samples were heat denatured for three minutes, immediately placed on ice, and electrophoretically separated at $4^{\circ} \mathrm{C}$ in $6 \%$ non-denaturing polyacrylamide gels which were run once with and once without $5 \%$ glycerol. The forward and reverse strands of PCR products corresponding to polymorphic mobility shifts were directly sequenced using the Thermo Sequenase radio- labelled terminator cycle sequencing kit (Amersham).

For the segregation analysis of the exon 13 polymorphism in the PBCRA and MCDR1 families, SSCP was carried out using the CleanGel-System (Pharmacia). ${ }^{22}$ To increase the sensitivity of the SSCP analysis, the exon 13 PCR fragment was digested with restriction enzyme NlaIV (New England Biolabs) before loading on a $15 \%$ non-denaturing polyacrylamide gel. This resulted in three fragments of 250, 210, and $140 \mathrm{bp}$ in size. Electrophoresis was done at $150 \mathrm{~V}, 6 \mathrm{~W}$ for 30 minutes followed by $550 \mathrm{~V}, 12 \mathrm{~W}$ for 60 minutes. The DNA was detected by silver staining as previously described. ${ }^{23}$

\section{Results}

MUTATIONAL ANALYSIS

For mutational analyses, DNA samples from one patient with late onset autosomal dominant Stargardt-like macular dystrophy, ${ }^{16}$ three patients diagnosed with North Carolina macular dystrophy, ${ }^{17}{ }^{18}$ and two patients with progressive bifocal chorioretinal atrophy from a single family ${ }^{14}$ were used (fig 2). The 17 exons of the IMPG1 gene were amplified using the
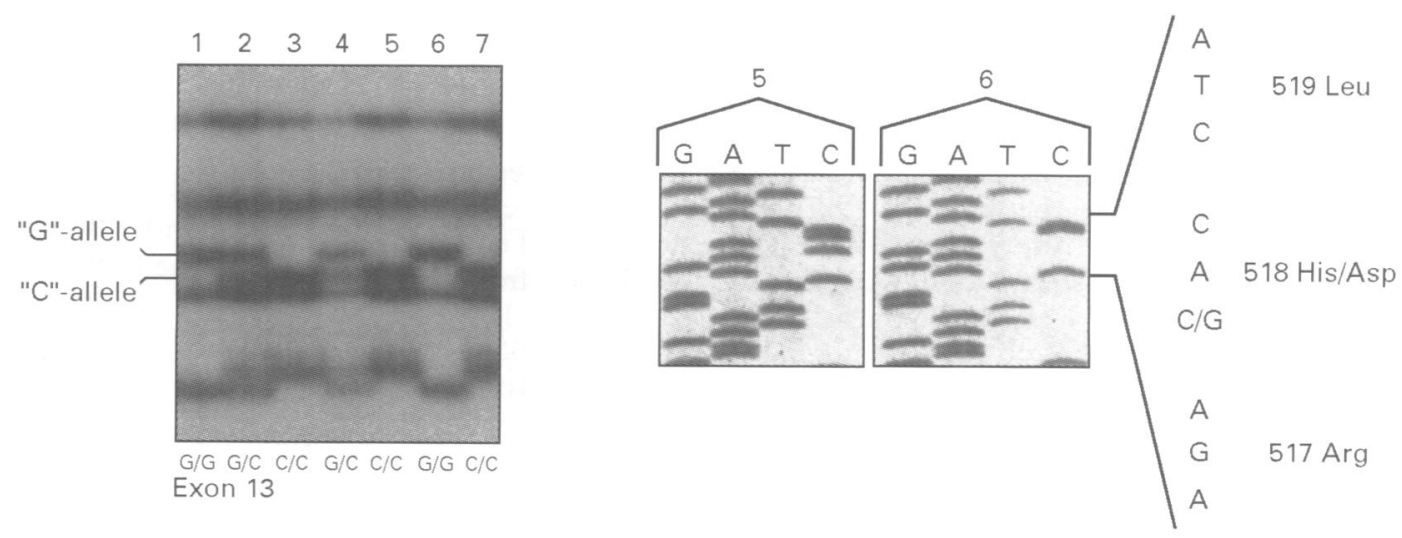

Figure 3 Single stranded conformational polymorphism (SSCP) in exon 13 of the IMPG1 gene. (A) SSCP of controls shows the polymorphic mobility shifts. (B) Sequencing of homozygous mobility shift as seen in controls 5 and 6 shows a $C$ to $G$ transversion at the first nucleotide of codon 518 resulting in a His/Asp protein polymorphism. 


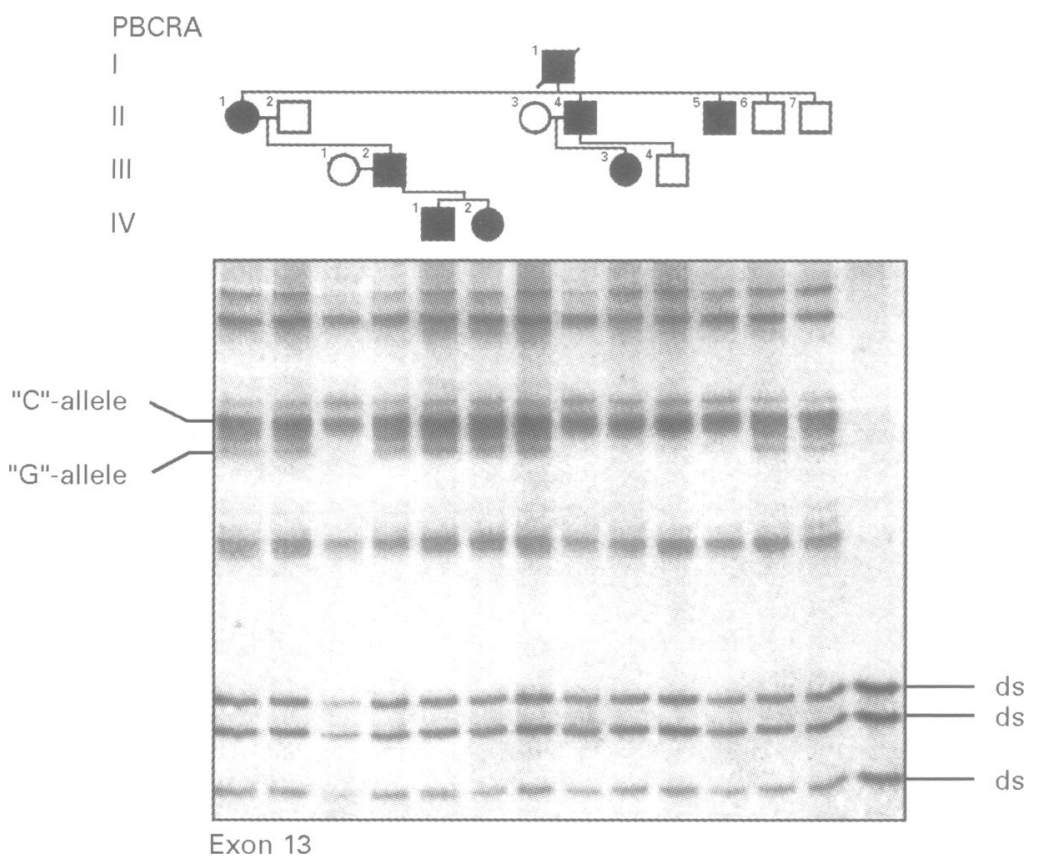

Figure 4 Segragation of $C / G$ polymorphism in a branch of the PBCRA family. Affected subjects II. 1, III.2, IV.1, and IV.2 are heterozygous for the "C" and " $G$ " allele, respectively. Affected subjects IV.1 and IV.2 have inherited the " $G$ " allele from their affected father III. 2 In contrast, affected subjects II. 4, II. 5, and III. 3 are homozygous for the " $C$ " allele. This analysis shows a recombination event between PBCRA and IMPG1 in this pedigree. $d s=$ double strand. autosomal dominant retinitis pigmentosa. ${ }^{24}$ Similarly, the peripherin/RDS gene was found to be mutated in autosomal dominant retinitis pigmentosa linked to chromosome $6 \mathrm{p} .^{25}$ Also, one form of Leber's congenital amaurosis was shown to be caused by mutations in the retinal specific guanylate cyclase on chromosome $17 \mathrm{p} .^{26}$ Colocalisation of Sorsby's fundus dystrophy (SFD) and the tissue inhibitor of metalloproteinases-3 (TIMP3) on chromosome 22q13-qter has led to the identification of TIMP3 as the gene underlying SFD pathology. ${ }^{27}$ Finally, functional aspects and chromosomal localisation to $1 \mathrm{p}$ has identified the rod photoreceptor cell specific ABC transporter as the gene causing autosomal recessive Stargardt's disease. ${ }^{28}$

Based on the chromosomal localisation of the interphotoreceptor matrix (IPM) proteoglycan gene, IMPG1, to $6 \mathrm{q} 13-\mathrm{q} 15^{12}$ and the potential role of IPM molecules in mediating interactions between retinal cells, the RPE and the choriocapillaris, ${ }^{89}$ we reasoned that IMPG1 represents an excellent candidate for chromosome $6 \mathrm{q}$ linked retinopathies, including autosomal dominant Stargardt-like macular dystrophy (STGD3), ${ }^{13}$ North Carolina macular dystrophy (MCDR1), ${ }^{15} 1718$ and progressive bifocal chorioretinal atrophy (PBCRA) ${ }^{14}$ (fig 1). However, SSCP analysis has not shown any aberrant mobility shifts in the affected subjects. Furthermore, using an intragenic IMPG1 polymorphism in exon 13 we have identified a recombination event between the IMPGI gene and the PBCRA locus.

Extended haplotype analysis has previously been performed in the PBCRA family using DNA markers spanning a genetic distance of approximately $50 \mathrm{cM}$ and has excluded a location of the PBCRA locus centromeric to D6S249 and telomeric to D6S268 in recombinant subject II. $^{14}$ (fig 2). The present IMPG1 allelotyping in the PBCRA family in combination with the previous findings in recombinant subject II. 1 excludes the IMPG1 gene from an interval flanked by markers D6S249 and D6S268. As this genetic interval contains the region harbouring the MCDR1 locus (fig 1), the PBCRA recombination data similarly exclude the IMPG1 gene as the cause of MCDR1 as well as PBCRA.

Autosomal dominant Stargardt-like macular dystrophy was analysed in a single affected member of a three generation family with clear autosomal dominant transmission of the Stargardt-like phenotype. ${ }^{16}$ Similarly to MCDR1 and PBCRA, SSCP analysis of the IMPG1 gene in the ADSTGD patient has not identified a disease causing mutation. However, it should be noted that such a mutation may have remained undetected owing to a well established detection sensitivity of the SSCP system for single base changes in the order of 75 to $85 \%$. Since DNA samples from additional members of the family were not available, we have not been able to perform genetic linkage analysis on this family. Therefore, the chromosomal position of the gene locus in this family is still unknown. However, it is well established that the autosomal
The application of the positional candidate approach has led to the identification of several genes associated with retinal dystrophies. For example, mutations in the rhodopsin gene have been shown to cause chromosome $3 \mathrm{q}$ linked 
dominant Stargardt-like macular dystrophies are genetically heterogeneous with at least two independent gene loci on chromosome $6 \mathrm{q}^{13}$ and chromosome $13 \mathrm{q} .{ }^{19}$ Thus, our results cannot rule out that mutations in the IMPG1 gene are involved in one form of autosomal dominant Stargardt-like macular dystrophy. To resolve this issue additional patients diagnosed with autosomal dominant Stargardt-like macular dystrophy need to be analysed. In particular, the mutational testing of IMPG1 in affected members of an autosomal dominant Stargardt-like macular dystrophy family previously mapped to proximal chromosome 6q (designated STGD3) ${ }^{13}$ will be useful.

This work was supported by grants from the Deutsche Forschungsgemeinschaft (DFG)

1 Collins FS. Positional cloning moves from perditional to traditional. Nat Genet 1995;9:347-50.

2 Kuehn MH, Hageman GS. Characterization of a cDNA encoding IPM150, a novel human interphotoreceptor encoding IPM150, a novel human interphotoreceptor matrix chondroitin 6-sulfate

3 Kuehn MH, Stone EM, Hageman GS. Molecular analyses of IPM150, a photoreceptor cell-specific proteoglycan. Invest Ophthalmol Vis $S_{c i}$ 1997:38.S599.

4 Johnson LV, Hageman GS, Blanks JC. Interphotoreceptor matrix domains ensheath vertebrate cone photoreceptor cells. Invest Ophthalmol Vis Sci 1986;27:129-35.

5 Hollyfield JG, Rayborn ME, Landers RA, Myers KM Insoluble interphotoreceptor matrix domains surround rod photoreceptors in the human retina. Exp Eye Res 1990;51: 107-10.

6 Fariss RN, Anderson DH, Fisher SK. Comparison of photoreceptor-specific matrix domains in the cat and monkey retinas. Exp Eye Res 1990;51:473-85.

7 Mieziewska KE, van Veen T, Murray JM, Aguirre GD. Rod and cone specific domains in the interphotoreceptor and cone specific domains in the inte

8 Hewitt AT. Extracellular matrix molecules: their importance in the structure and function of the retina. In: Adler R, Farin the structure and function of the retina. In: Adler R, Far-
ber D, eds. The retina: a model for cell biology studies. Part II ber D, eds. The retina: a model for cell biology
London: Academic Press, 1986:170-201.

9 London: Academic Press, 1986:170-201. function of the retinal interphotoreceptor matrix. In Osborne N, Chader J, eds. Progress in retinal research. Vol 10. Oxford: Pergamon Press, 1991:207-49.

10 Lazarus HS, Hageman GS. Xyloside-induced disruption of interphotoreceptor matrix proteoglycans results in retinal detachment. Invest Ophthalmol Vis Sci 1992;33:364-76. 11 Hageman GS, Marmor MF, Yao XY, Johnson LV. The sion. Arch Ophthalmol 1995;113:655-60.
12 Felbor U, Gehrig A, Sauer CG, et al. Genomic organization and chromosomal localization of the interphotoreceptor matrix proteoglycan-1 (IMPG1) gene: a candidate for $6 \mathrm{q}$ linked retinopathies. Cytogenet Cell Genet (in press).

13 Stone EM, Nichols BE, Kimura AE, Weingeist TA, Drack A, Sheffield VC. Clinical features of a Stargardt-like dominant progressive macular dystrophy with genetic linkage to chromosome 6q. Arch Ophthalmol 1994;112:765-72.

14 Kelsell RE, Godley BF, Evans K, et al. Localization of the gene for progressive bifocal chorioretinal atrophy $(P B C R A)$ to chromosome 6q. Hum Mol Genet 1995;4:1653-6.

15 Small KW, Weber JL, Roses A, Lennon F, Vance JM Pericak-Vance MA. North Carolina macular dystrophy is assigned to chromosome 6 . Genomics 1992;13:681-5.

16 Lopez PF, Maumenee IH, de la Cruz Z, Green WR. Autosomal-dominant fundus flavimaculatus. Clinicopathologic correlation. Ophthalmology 1990;97:798-809.

17 Pauleikhoff D, Sauer CG, Müller CR, Radermacher M, Merz A, Weber BHF. Clinical and genetic evidence for autosomal dominant North Carolina macular dystrophy in a German family. Am 7 Ophthalmol 1997;124:412-15.

18 Sauer CG, Schworm HD, Ulbig M, et al. An ancestral core haplotype defines the critical region harbouring the North Carolina macular dystrophy gene (MCDR1). F Med Genet 1997;34:961-6.

19 Zhang K, Bither PP, Park R, Donoso LA, Seidman JG, Seidman CE. A dominant Stargardt's macular dystrophy locus maps to chromosome 13q34. Arch Ophthalmol 1994;112: 759-64

20 Douglas AA, Waheed I, Wyse CT. Progressive bifocal chorio-retinal atrophy. A rare familial disease of the eyes. $B r$ f Ophthalmol 1968;52:742-51.

21 Godley BF, Tiffin PA, Evans K, Kelsell RE, Hunt DM, Bird AC. Clinical features of progressive bifocal chorioretinal atrophy: a retinal dystrophy linked to chromosome $6 \mathrm{q}$. Ophthalmology 1996;103:893-8.

22 Ainsworth PJ, Surh LC, Coulter-Mackie MB. Diagnostic single strand conformational polymorphism, (SSCP): a simplified non-radioisotopic method as applied to Tay-Sachs B1 variant. Nucleic Acids Res 1991;19:405-6.

23 Bassam BJ, Caetano-Anolles G, Gresshoff PM. Fast and sensitive silver staining of DNA in polyacrylamide gels. Anal Biochem 1991;196:80-3.

24 Dryja TP, McGee TL, Reichel E, et al. A point mutation of the rhodopsin gene in one form of retinitis pigmentosa. Nature 1990;343:364-6.

25 Farrar GJ, Kenna P, Jordan-Fajendra KS, et al. A three-base-pair deletion in the peripherin-RDS gene in one form of retinitis pigmentosa. Nature 1991;354:478-80.

26 Perrault I, Rozet JM, Calvas P, et al. Retinal-specific guanylate cyclase gene mutations in Leber's congenital amaurosis. Nat Genet 1996;14:461-4.

27 Weber BHF, Vogt G, Pruett RC, Stöhr H, Felbor U. Mutations in the tissue inhibitor of metalloproteinases-3 (TIMP-3) in patients with Sorsby's fundus dystrophy. Nat Genet 1994;8:352-6.

28 Allikmets R, Singh N, Sun $\mathrm{H}$, et al. A photoreceptor cell specific ATP binding transporter gene (ABCR) is mutated in recessive Stargardt macular dystrophy. Nat Genet $1997 ; 15: 236-46$ 